\title{
Is WHODAS 2.0 Useful for Colorectal Cancer Survivors?
}

\author{
Hyun Haeng Lee, MD ${ }^{1}$, Eun-Kyoung Shin, $\mathrm{PhD}^{2}$, Hyung-Ilk Shin, MD, PhD ${ }^{1}$, Eun Joo Yang, MD, PhD ${ }^{3}$ \\ ${ }^{1}$ Department of Rehabilitation Medicine, Seoul National University Hospital, Seoul; \\ ${ }^{2}$ Department of Social Welfare, Dankook University, Yongin; \\ ${ }^{3}$ Department of Rehabilitation Medicine, Bundang Seoul University Hospital, Seongnam, Korea
}

\begin{abstract}
Objective To compare the disability level of colorectal cancer survivors with and without stoma by using the Korean version of the 12-item, interview-administered World Health Organization Disability Assessment Schedule 2.0 (Korean version of WHODAS 2.0).

Methods This is a multicenter (five tertiary university hospitals and the Korea Ostomy Association) and crosssectional survey. Colorectal cancer survivors with and without stoma were interviewed. Survey measured disability level using the Korean version of WHODAS 2.0 and health-related quality of life using the SF-36.

Results A significant difference was observed between patients with and without a stoma in two subdomains: getting around (31.1 vs. 20.3; $\mathrm{p}=0.013$ ) and participation in society (32.3 vs. 22.2; $\mathrm{p}=0.028$ ). After adjusting for age, gender, and time since surgery, having a stoma was associated with severe to extreme disabilities in participation $(\mathrm{OR}=2.72, \mathrm{p}=0.045)$. The Korean version of WHODAS 2.0 showed satisfactory internal consistency $(\mathrm{r}=0.96)$ and convergent validity.

Conclusion Patients with stoma participated less in society than those without stoma. The Korean version of WHODAS 2.0 is a reliable and valid instrument for measuring disability in Korean colorectal cancer patients.
\end{abstract}

Keywords International Classification of Functioning, Disability and Health (ICF), Colorectal neoplasms, Surgical stomas, Quality of life

\section{INTRODUCTION}

Colorectal cancer is one of the most common types of solid cancers, and survival rates are improving in all patient groups [1]. Increases in life expectancy for colorectal patients have emphasized the need for minimizing disability and improving quality of life (QoL) [1]. Previous research suggests that a return or maintenance of health, functional capacity, and emotional well-being are highly valued goals of patients [2]. To optimize interventions

Received September 21, 2016; Accepted October 20, 2016

Corresponding author: Eun Joo Yang

Department of Rehabilitation Medicine, Bundang Seoul University Hospital, 82 Gumi-ro 173beon-gil, Bundang-gu, Seongnam 13620, Korea. Tel: +8231-793-7734, Fax: +82-31-787-4056, E-mail: graceloves@gmail.com

ORCID: Hyun Haeng Lee (http://orcid.org/0000-0001-6666-6284); Eun-Kyoung Shin (http://orcid.org/0000-0003-0025-5065); Hyung-Ik Shin (http:// orcid.org/0000-0001-8805-3104); Eun Joo Yang (http://orcid.org/0000-0003-2417-2416).

(c) This is an open-access article distributed under the terms of the Creative Commons Attribution Non-Commercial License (http://creativecommons.org/ licenses/by-nc/4.0) which permits unrestricted noncommercial use, distribution, and reproduction in any medium, provided the original work is properly cited. Copyright (C) 2017 by Korean Academy of Rehabilitation Medicine 
aimed toward maintaining optimal functioning and minimizing disability, a proper understanding of patients' disabilities is required. The World Health Organization (WHO) International Classification of Functioning, Disability and Health classifies disability (ICF) as "difficulties in any area of functioning as they relate to environmental and personal factors."

The disabilities experienced by colorectal cancer survivors may differ according to the presence of a colostomy. Many studies suggest that patients with a stoma have poorer, health-related QoL than patients without stoma $[3,4]$. However, after a radical resection of colorectal cancer without a colostomy, patients with very low anastomoses are prone to developing frequent sexual and urinary dysfunction and fecal incontinence [5]. One study found QoL to be similar in patients with intersphincteric resection and patients with abdominoperineal resection plus perineal colostomy [6]. A recent Cochrane review found that patients with a stoma did not have poorer QoL than patients undergoing anterior resection [7]. Thus, research assessing the impact of stomas on disability can help to increase awareness among colorectal cancer survivors and guide the development and planning of healthcare services for these patients.

Although there have been some studies on the overall QoL of colorectal cancer survivors, structured outcome studies on disability experienced by colorectal cancer survivors are still scarce. An instrument used to measure disability should include, but not be limited to, an assessment of physical functioning or QoL. Furthermore, a study should ideally go beyond focusing specifically on the presence of symptoms. Studies should also assess the impact of symptoms on the patient's life, in the dimensions of cognition, mobility, self-care, getting along, life activity, and participation [8].

The WHO Disability Assessment Schedule 2.0 (WHODAS 2.0) measures disability cross-culturally, in the aged, and for disease-related states [8]. The WHODAS 2.0 is a self-assessment tool for measuring the limitations in daily activities, independently from a diagnosis. Its selfevaluation tool is a fundamental element compared to the questionnaires traditionally used for assessing disability. Unlike with self-evaluation tools, questionnaires are likely to be influenced by the perspectives of the caregivers or physicians who compile them.

The biopsychosocial model of WHODAS 2.0 is an in- novative method for conceptualizing disability and functioning. This model absolutely prioritizes the subjective perspective, compared to any other etiopathological evaluation tool. The psychometric properties of the WHODAS 2.0 have been studied in different community populations $[9,10]$, and for various conditions including depression [11] and breast cancer [12]. However, there are no reports evaluating its use in colorectal cancer patients or in other cancer care.

Therefore, we aimed to assess the disability of Korean colorectal cancer survivors by using the Korean version of WHODAS 2.0. We confirmed the reliability and validity of using this version to study Korean colorectal cancer survivors with disabilities, and then compared results between patients with and without a stoma.

\section{MATERIALS AND METHODS}

\section{Participants and recruitment}

We recruited colorectal cancer patients with stoma from the Korea Ostomy Association (KOA, http://www. ostomy.or.kr/). We completed recruitment by telephone or sign-up through the aforementioned website. We obtained informed consent from all patients.

Two interviewers with extensive experience ( $\geq 3$ years) conducted the interviews. We provided the interviewers with training on how to assess disabilities. They conducted face-to-face interviews at the KOA office.

Inclusion criteria were (1) people with a stoma aged 20 to 64 years, (2) living in a community, and (3) persons without mental illness. The survey data included the characteristics of respondents' disabilities and details of their health conditions.

We recruited colorectal cancer survivors without stoma from five tertiary hospitals in Korea. We confirmed potential participants' diagnoses by telephone prior to requesting their participation in the study. We then evaluated patients who met the criteria in outpatient clinics. All participants provided written, informed consent.

The Institutional Review Board of all the related university hospitals approved this study's protocol. This included approval from Seoul National University Bundang Hospital (IRB No. B-1110-138-008). 
Instrument for disability measurement and quality of life

We used the Korean version of the 12-item intervieweradministered WHODAS 2.0 [13] to evaluate an individual's level of functioning. Each item was linked to the ICF code. The ICF code scores as follows: none $=1$, mild $=2$, moderate $=3$, severe $=4$, and extreme $/$ cannot do $=5$. We totaled the scores from items within a category to obtain a raw domain score [8]. The domain scores were then combined to give a global disability score, with higher scores indicating higher levels of disability.

To enable easy comparison, raw global disability scores and each domain score were then transformed to a 0-100 scale using the complex WHODAS method of scoring [14]. We categorized the global disability scores according to the ICF severity ranges (no problem, 0-4; mild disability, 5-24; moderate disability, 25-49; severe/extreme disability, 50-100). We considered a disability score of greater than or equal to 25 to indicate 'disability' based on the WHODAS ICF.

We chose the WHODAS 2.0 as our preference to other traditional instruments for several reasons. First, the WHODAS 2.0 is an ICF-based instrument used in epidemiological studies and clinical settings. Second, the WHODAS 2.0 provides ICF assessments of contextual, physical, and social environmental factors that impact disability and effect health conditions. Third, the WHODAS 2.0 takes into account facets of disability that are related to patient requirements for support, such as their ability to communicate with others and maintain an active social life. Other scales, such as the extended Katz and Lawson scales, focus on activities of daily living, but overlook these other important facets [15].

We used the SF-36 (Medical Outcome Trust, Boston, MA, USA), a multipurpose short-form health survey with only 36 questions, to confirm the validity of the Korean version of WHODAS 2.0 in colorectal cancer survivors. This measure yields summary scores for physical and mental health, as well as eight subscales. These subscales are (1) physical functioning, (2) role limitations resulting from physical problems, (3) bodily pain, (4) general health, (5) vitality, (6) social function, (7) role limitations resulting from emotional problems, and (8) mental health.

The SF-36 has the advantage of norm-based scoring ( $m e a n=50, \mathrm{SD}=10$ ) based on very large and diverse popu- lations, including individuals with back pain. The SF-36 has also been extensively translated. With rare exceptions, the published reliability statistics have exceeded the minimum standard of 0.70 recommended for such measures, and most have exceeded 0.80 [16].

\section{Demographic and disease characteristics}

Demographic characteristics included the following: age, gender, marital status (living with/without spouse), education level (no education or elementary school graduate/middle or high school graduate/university graduate or higher), residential area (rural/urban), and monthly individual income (dichotomized into low and high, split at US $\$ 1,779$, personal contribution for medical services covered by the Korean national insurance system and limited to $14 \%$ of whole cost). Individuals who were legally married or cohabiting were considered to have a spouse; single, divorced, or separated individuals were categorized as being without a spouse. Time since surgery was also recorded.

\section{Analysis}

We assessed internal consistency using Cronbach's $\alpha$ coefficient, which summarizes interim correlations among items in a scale. This statistic evaluates the items to determine whether they are measuring the same construct or are redundant. The evaluation thus indicates which items can be discarded to improve the homogeneity of the scale [17].

We assessed the validity (or accuracy) of WHODAS 2.0 according to the following guidelines. (1) Content validity: WHODAS 2.0 was developed and has been extensively validated as a responsive measure of health-related disability, as defined by the ICF. (2) Convergent validity: we compared the total and subscale scores of the WHODAS 2.0 to the total and subscale SF-36 scales. We conducted sensitivity analyses to explore whether patients' age modified correlations. In general, correlation coefficients $<0.3,0.3-0.49$, and $>0.5$ represent small, medium, and large correlations, respectively.

We studied the capacity of the WHODAS 2.0 to differentiate between groups. We calculated the magnitude of the differences between WHODAS 2.0 scores as Cohen's $d$ by subtracting the mean scores in both groups, and divided by the pooled standard deviation.

We completed Student t-test and chi-squared tests to 
identify any differences between the two study groups with regard to demographic, socioeconomic, psychological variables, and health-related behavior patterns. We also evaluated these test results to see if there was a more understandable measurement of reported problems in each of the WHODAS 2.0 domains. We performed a univariate logistic regression analysis to assess the relationship between disability (severe or complete disability in each WHODAS 2.0 domain) and clinical or demographic data. Variables that showed a 'significant association' or were of 'considerable theoretical relevance' were entered into the multivariate logistic regression analysis using the conditional elimination method.

We analyzed the determinants of disability for each domain of the Korean version of WHODAS 2.0 by performing a multivariate logistic regression analysis. For this analysis, we divided the four groups into two-the presence of severe disability group and the complete disability group. These two groups were the dependent variables. We excluded co-variables that had co-linearity from the multivariate analysis.

We expressed the distribution of scores on the scale as percentage of subjects, with a minimum or maximum possible score. We conducted our analyses using SPSS software ver. 19.0 (SPSS, Inc., Chicago, IL, USA). We considered $\mathrm{p}<0.05$ as statistically significant.

\section{RESULTS}

\section{Participants' characteristics}

We included a total of 132 patients in this study. Table 1 displays patients' sociodemographic data and clinical characteristics. As seen in Table 1, there were no differences in age, gender, marital, and income status between participants with or without a stoma. However, patients with a stoma were more highly educated, and less likely to be employed than those without a stoma. Patients with a stoma had a significantly longer post-surgery period

Table 1. Characteristics of colorectal cancer patients with and without a stoma

\begin{tabular}{|c|c|c|c|c|}
\hline & $\begin{array}{c}\text { Total } \\
(n=132)\end{array}$ & $\begin{array}{l}\text { With stoma } \\
\quad(n=66)\end{array}$ & $\begin{array}{l}\text { Without stoma } \\
\qquad(n=66)\end{array}$ & p-value \\
\hline \multicolumn{5}{|l|}{ Demographic factors } \\
\hline Male & $86(65.2)$ & $44(66.7)$ & $42(63.6)$ & 0.475 \\
\hline Female & $46(34.8)$ & $22(33.3)$ & $24(36.4)$ & \\
\hline Age (yr) & $62.1 \pm 9.7(36-83)$ & $62.6 \pm 9.2(44-83)$ & $61.5 \pm 9.6(36-79)$ & 0.504 \\
\hline \multicolumn{5}{|c|}{ Marital (living with spouse) } \\
\hline Yes & $106(80.3)$ & $55(83.3)$ & $51(77.3)$ & 0.329 \\
\hline No & 25 (18.9) & $11(16.7)$ & $14(21.2)$ & \\
\hline No response & $1(0.8)$ & $0(0.0)$ & $1(1.5)$ & \\
\hline \multicolumn{5}{|l|}{ Income (US\$) } \\
\hline$<1,779$ & $62(47.0)$ & $35(53.0)$ & $27(40.9)$ & 0.144 \\
\hline$\geq 1,779$ & $68(51.5)$ & $31(47.0)$ & $37(56.1)$ & \\
\hline No response & $2(1.5)$ & $0(0.0)$ & $2(3.0)$ & \\
\hline \multicolumn{5}{|l|}{ Education } \\
\hline$\leq$ Middle school & $59(44.7)$ & $16(24.2)$ & $43(65.2)$ & $<0.001^{*}$ \\
\hline$\geq$ High school & $72(54.5)$ & $50(75.8)$ & $22(33.3)$ & \\
\hline No response & $1(0.8)$ & $0(0.0)$ & $1(1.5)$ & \\
\hline \multicolumn{5}{|l|}{ Occupation } \\
\hline Yes & $66(50.0)$ & $20(30.3)$ & $46(69.7)$ & $<0.001^{*}$ \\
\hline No & $65(49.2)$ & $46(69.7)$ & $19(28.8)$ & \\
\hline No response & $1(0.8)$ & $0(0.0)$ & $1(1.5)$ & \\
\hline Time since surgery (yr) & $7.3 \pm 4.2(0-27)$ & $11.8 \pm 6.9(0-27)$ & $2.7 \pm 2.6(0-13)$ & $<0.001^{*}$ \\
\hline
\end{tabular}

Values are presented as number (\%) or mean \pm standard deviation (range). ${ }^{*} \mathrm{p}<0.001$. 
(11.8 years vs. 2.7 years; $\mathrm{p}<0.001$ ).

For WHODAS 2.0, the number of missing values per item ranged from $0.0 \%$ to $1.5 \%$. There was one exception with S12, "Your day-to-day work/school," which had missing values of $30.3 \%$. Ceiling effects were most pronounced in the "understanding and communicating" and "getting along with people" domains, with more than $50 \%$ of replies falling into the top response category.

We report the global disability score and Korean version of WHODAS 2.0 domain scores as the mean \pm SD. The Korean version of WHODAS 2.0 total score was $46.0 \pm 18.3$. The greatest impairments were observed for "participation in society" (52.9 \pm 25.0$)$ and "getting along with people" (52.7 \pm 26.7$)$. With these two impairments, $47 \%$ and $48.5 \%$ of patients, respectively, had scores of $\geq 50$ (Table 2 ).

\section{Reliability}

The internal consistency of the Korean version of WHODAS 2.0 total score was high (Cronbach's $\alpha=0.96$ ). Cronbach's $\alpha$ for the Korean version of WHODAS 2.0 subscales also showed strong internal consistency except with selfcare $(\alpha=0.52)$ (Table 2).

\section{Content and construct validity}

Results from the analysis of convergent validity of the Korean version of WHODAS 2.0 showed correlations of moderate to large size (Table 3). The physical component summary and mental component summary of the SF-36 showed convergent validity with the Korean version of WHODAS 2.0 subscales and total scores.

\section{Comparison of functioning between colorectal cancer survivors with and without stoma}

The analysis of the capacity of the Korean version of WHODAS 2.0 to differentiate between stoma groups indicated that the Korean version of WHODAS 2.0 scores did not differ significantly: with stoma $(n=66)$ vs. without stoma ( $\mathrm{n}=66) ; 19.5 \pm 18.3$ vs. $22.5 \pm 23.3$; $\mathrm{p}=0.421$.

Patients with a stoma reported significantly higher scores for getting around and participation domains,

Table 2. Distribution and reliability of the WHODAS $2.0(\mathrm{n}=132)$

\begin{tabular}{lccccc}
\hline \multicolumn{1}{c}{ Domain } & $\begin{array}{c}\text { Observed } \\
\text { range }\end{array}$ & $\begin{array}{c}\text { Transformed } \\
\text { score } \\
\mathbf{( 0 - 1 0 0 )}\end{array}$ & $\begin{array}{c}\text { Missing } \\
\text { domain (\%) }\end{array}$ & $\begin{array}{c}\text { Proportion with } \\
\text { transformed } \\
\text { score } \mathbf{5 0} \text { (\%) }\end{array}$ & $\begin{array}{c}\text { Internal } \\
\text { consistency } \\
\text { (Cronbach's } \boldsymbol{\alpha})\end{array}$ \\
\hline Understanding and communicating & $0.0-87.5$ & $39.6 \pm 17.4$ & 0.3 & 27.3 & 0.91 \\
Getting around & $0.0-90.0$ & $41.5 \pm 18.4$ & 0.6 & 33.3 & 0.87 \\
Self-care & $0.0-100.0$ & $44.2 \pm 25.9$ & 0.8 & 34.8 & 0.52 \\
Getting along with people & $0.0-100.0$ & $52.7 \pm 26.7$ & 0 & 47.0 & 0.73 \\
Household activities & $0.0-100.0$ & $45.2 \pm 24.8$ & 15.2 & 37.9 & 0.94 \\
Participation in society & $0.0-100.0$ & $52.9 \pm 25.0$ & 0.2 & 48.5 & 0.94 \\
\hline Global disability & $0.0-100.0$ & $46.0 \pm 18.3$ & 3.6 & 37.9 & 0.96 \\
\hline
\end{tabular}

Values are presented as mean \pm standard deviation.

WHODAS 2.0, World Health Organization Disability Assessment Schedule 2.0.

Table 3. Spearman correlation coefficients between the WHODAS 2.0 and SF-36

\begin{tabular}{|c|c|c|c|c|c|c|c|}
\hline & \multicolumn{7}{|c|}{ WHODAS 2.0 subscales } \\
\hline & \multicolumn{3}{|c|}{ Activity limitation } & \multicolumn{4}{|c|}{$\begin{array}{l}\text { Participation restrictions } \\
\end{array}$} \\
\hline & $\begin{array}{l}\text { Understanding and } \\
\text { communicating }\end{array}$ & $\begin{array}{l}\text { Getting } \\
\text { around }\end{array}$ & Self-care & $\begin{array}{l}\text { Getting along } \\
\text { with people }\end{array}$ & $\begin{array}{c}\text { Household } \\
\text { activities }\end{array}$ & $\begin{array}{l}\text { Participation } \\
\text { in society }\end{array}$ & Total \\
\hline \multicolumn{8}{|l|}{ SF-36 } \\
\hline Physical health & $-0.611^{*}$ & $-0.766^{*}$ & $-0.685^{*}$ & $-0.667^{*}$ & $-0.425^{*}$ & $-0.699^{*}$ & $-0.775^{*}$ \\
\hline Mental health & $-0.698^{*}$ & $-0.746^{*}$ & $-0.626^{*}$ & $-0.693^{*}$ & $-0.442^{*}$ & $-0.765^{*}$ & $-0.788^{*}$ \\
\hline Total & $-0.684^{*}$ & $-0.792^{*}$ & $-0.664^{*}$ & $-0.693^{*}$ & $-0.422^{*}$ & $-0.756^{*}$ & $-0.799 *$ \\
\hline
\end{tabular}

WHODAS 2.0, World Health Organization Disability Assessment Schedule 2.0.

${ }^{*} \mathrm{p}<0.001$. 


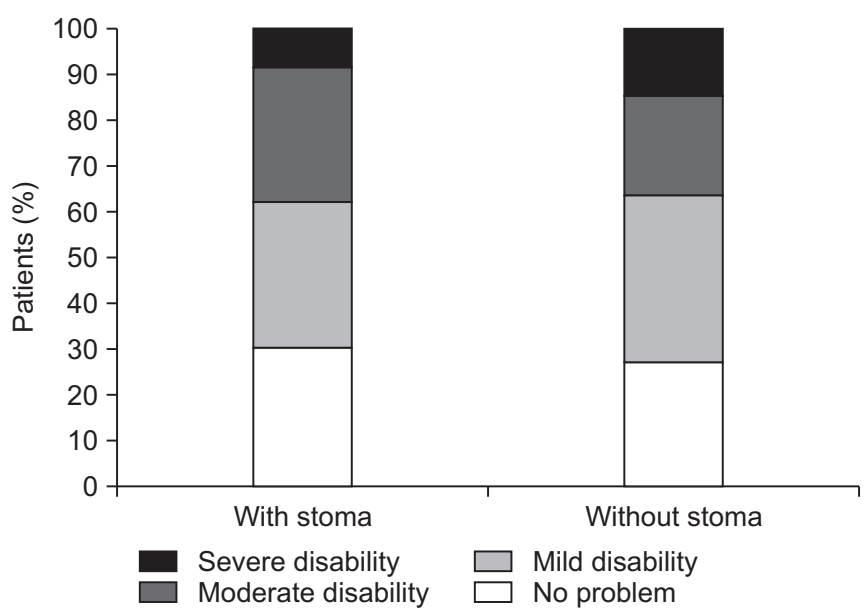

Fig. 1. The World Health Organization Disability Assessment Schedule 2.0 (WHODAS 2.0) scores (mean) in relation to stoma status.

compared to patients without a stoma (Fig. 1). Patients with stoma vs. without stoma had a higher prevalence of severe/extreme disability in getting around $(24.2 \%$ vs. $19.7 \%)$; getting along with people (34.3\% vs. $19.7 \%)$; life activity (39.4\% vs. $25.8 \%$ ); and participation in society (33.3\% vs. $15.2 \%$ ) (Fig. 2). We scored these categories using the ICF severity ranges.

After adjusting for control variables, such as gender, age, and time since surgery, patients with stoma had more severe levels of disability in the participation domain ( $\beta$ coefficient $=0.09,95 \%$ confidence interval $[\mathrm{CI}]$, 0.01-4.21) (Table 4). When comparing each ICF category for the two study groups, patients with a stoma were more emotionally affected by their disease $(\mathrm{OR}=2.57$, 95\% CI, 1.03-7.32) but had fewer problems in concentrating on doing something for 10 minutes $(\mathrm{OR}=0.22,95 \%$ CI, 0.06-0.82). This was the case, even after adjusting for gender, age, and time since surgery (Table 5).

\section{DISCUSSION}

We found that colorectal cancer survivors had low levels of functioning, with adjusted means for each domain of the Korean version of WHODAS 2.0 scoring greater than 10. According to the study, which surveyed over 65,000 respondents, the general population has adjusted means for each domain and general scores lower than 10 [8].

A different study examined how cancer and treatment sequelae combine with comorbidities to influence physi- cal functioning and activity participation restrictions, including in older colorectal cancer survivors [18]. With regard to functional status, the data indicate that cancerrelated factors play a role in functional limitations, and have a modest indirect effect on participants' perceptions of their functioning.

Here, we observed a significant relationship between the presence of a stoma and functional issues, particularly with respect to restriction of participation in activities. After comparing each ICF category, we found that living with a stoma had a more significantly negative impact on patients' emotional well being.

This finding is supported by previous studies. One study disclosed that despite a reportedly good QoL, patients with stoma had difficulties with their sexuality and body image (e.g., difficulty with clothing, feeling unattractive) [19]. Patients also had practical concerns related to the stoma itself, such as finding privacy to empty the pouch and problems with leakage [19]. Another study found that older patients with a stoma had comparable QoL to that of older patients without a stoma, but the stoma had a negative impact on sexual activity [20].

Given the high incidence of emotional problems and the impact on function (including sexual functioning), the negative emotional impact of living with a stoma should be addressed whenever possible. For instance, we could provide colorectal cancer survivors with specific psychosocial and clinical support.

Our study shows that the impact of the stoma on general functioning in colorectal cancer patients is low regardless of sex and age. Similar findings were recently reported in a large meta-analysis and Cochrane review when considering QoL [7]. Other recent studies have shown that factors like gender and post-operative complications, but not the stoma, impact the QoL of colorectal cancer patients [21,22].

In our study, patients with a stoma had a lower risk of severe to extreme disability in concentrating on doing a task for 10 minutes. An external sphincter-saving procedure was recently used to preserve the sphincter, and therefore avoid permanent colostomy. However, this procedure can cause severe bowel dysfunction resulting in incontinence, urgency, and frequent bowel movements, which have a negative impact on QoL. Our study did not directly assess incontinence or urgency symptoms; however, we postulate that patients with a stoma may have 

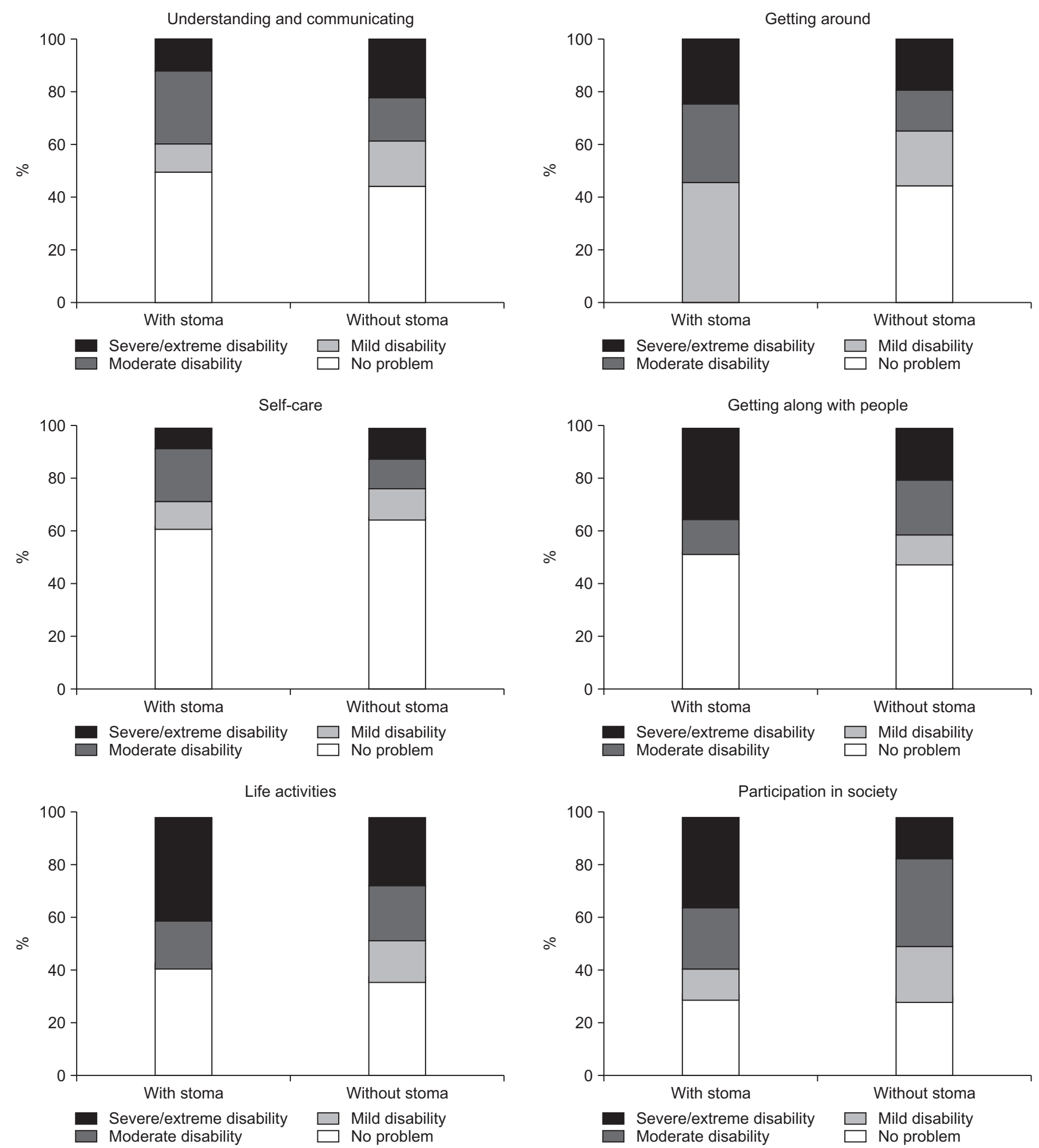

Fig. 2. Prevalence of different ICF categories by The World Health Organization Disability Assessment Schedule 2.0 (WHODAS 2.0) domain score for colorectal cancer patients with and without a stoma. ICF, International Classification of Functioning, Disability and Health. 
Table 4. Comparative disability pattern by WHODAS 2.0 domain in colorectal cancer patients with stoma compared with those without stoma

\begin{tabular}{lccc}
\hline & Model 1 & Model 2 & Model 3 \\
\hline Cognition & $0.42(0.15-1.18)$ & $0.39(0.14-1.14)$ & $0.15(0.03-1.18)$ \\
\hline Getting around & $1.26(0.52-3.06)$ & $1.24(0.49-3.13)$ & $0.85(0.24-3.01)$ \\
\hline Self-care & $0.43(0.11-1.64)$ & $0.40(0.10-1.57)$ & $1.26(0.23-7.05)$ \\
\hline Getting-along & $1.86(0.79-4.37)$ & $1.85(0.78-4.37)$ & $1.43(0.47-4.43)$ \\
\hline Life activities & $1.58(0.71-3.48)$ & $1.56(0.69-3.52)$ & $1.53(0.52-4.46)$ \\
\hline Participation & $2.74(1.11-6.79)$ & $2.73(1.10-6.79)$ & $2.72(1.11-6.77)$ \\
\hline Disability & $0.45(0.13-1.55)$ & $0.40(0.11-1.50)$ & $0.83(0.15-4.63)$ \\
\hline
\end{tabular}

WHODAS 2.0, World Health Organization Disability Assessment Schedule 2.0; Model 1, adjusted for sex; Model 2, adjusted for sex and age; Model 3, adjusted for sex, age, and time since surgery.

Table 5. Comparative disability pattern by each ICF categories of colorectal cancer patients with stoma compared to those without stoma

\begin{tabular}{lccc}
\hline & Model 1 & Model 2 & Model 3 \\
\hline d4154 standing & $0.74(0.33-1.66)$ & $0.71(0.31-1.63)$ & $0.64(0.21-1.93)$ \\
\hline d640 household & $1.23(0.59-2.58)$ & $1.23(0.58-2.57)$ & $0.98(0.37-2.59)$ \\
d1551 learning & $0.39(0.16-1.01)$ & $0.36(0.14-1.01)$ & $0.19(0.05-1.02)$ \\
d910 joining community & $0.45(0.21-1.02)$ & $0.45(0.21-1.03)$ & $0.36(0.14-1.03)$ \\
\hline d152 emotionally affected & $2.04(1.01-4.64)$ & $2.07(1.03-4.70)$ & $2.57(1.03-7.32)$ \\
d160 concentrating & $0.24(0.09-0.65)$ & $0.23(0.08-0.63)$ & $0.22(0.06-0.82)$ \\
\hline d4501 walking & $1.09(0.44-2.67)$ & $1.07(0.43-2.65)$ & $0.94(0.29-3.07)$ \\
\hline d5101 washing & $1.19(0.54-2.62)$ & $1.18(0.53-2.61)$ & $0.95(0.33-2.72)$ \\
d540 dressing & $0.36(0.10-1.29)$ & $0.36(0.10-1.29)$ & $1.41(0.29-6.91)$ \\
d730 dealing do not know & $1.47(0.69-3.13)$ & $1.47(0.69-3.14)$ & $1.49(0.55-4.03)$ \\
\hline d7500 maintaining friendship & $0.48(0.22-1.03)$ & $0.48(0.22-1.03)$ & $0.25(0.09-1.02)$ \\
\hline d850 work & $0.55(0.26-1.16)$ & $0.54(0.26-1.15)$ & $0.54(0.20-1.43)$ \\
\hline
\end{tabular}

ICF, International Classification of Functioning, Disability and Health; Model 1, adjusted for sex; Model 2, adjusted for sex and age; Model 3, adjusted for sex, age, and time since surgery.

fewer problems concentrating than patients without a stoma, because they might be less distracted by frequent symptoms and bowel movements.

Our results showed internal consistency reliability for the Korean version of WHODAS 2.0 subscale of $0.52-0.94$, with a Cronbach's $\alpha$ value of 0.96 for the Korean version of WHODAS 2.0 total score. All the subscales, with the exception of self-care, were above the standard proposed for group comparisons (0.7) [23]. Internal consistency was similar to previous studies of specific patient populations (e.g., patients with osteoarthritis, low back pain, stroke, and depression) [24]. Our study demonstrated satisfactory reliability for the Korean version of WHODAS 2.0 in colorectal cancer patients. We found strong support for convergent validation of the Korean version of WHODAS
2.0, estimated by correlation coefficients between Korean version of WHODAS 2.0 and SF-36 scores. Similarly, large correlation coefficients have been observed between the total WHODAS 2.0 score and the composite SF-36 score in patients with depression and back pain [25].

There are four important limitations to consider when interpreting the results of our study. First, one limitation was selection bias, which restricts the applicability of these findings to similar groups of colorectal cancer survivors. Our study sample may not be representative of the general population of colorectal cancer survivors, since our sample accrued from a limited group of academic centers. Second, we recruited the patients according to the existence of stoma, which could result in selection bias. For instance, we made a comparison between the 
groups without adjusting the different distribution of "Time since surgery." Moreover, the stage of cancer could also affect the disability of patients. However, we could not adjust the stage of colorectal cancer due to the lack of information. Third, we did not make a comparison with the general population without cancer. Fourth, in this cross-sectional study, we were not able to assess functional changes before and after treatment; thus, it was not possible to determine how functioning before cancer treatment influenced functioning after treatment. Additional prospective studies are needed to obtain a greater understanding of the relative functional disability of colorectal cancer survivors.

Despite the aforementioned limitations, this study confirms that the Korean version of WHODAS 2.0 possesses very high internal consistency reliability and good convergent validity in Korean colorectal cancer survivors. We found that patients with a stoma participated less in society and were more affected emotionally than those without a stoma. These findings provide useful information for clinicians preparing and informing patients for cancer treatment by providing them with an understanding of the potential outcome options. This information can also serve as a basis for potential educational intervention for rehabilitation and emotional supportive care needs among colorectal cancer survivors. The extensive range of postoperative outcomes renders it crucial for potential surgical patients to be fully educated in the spectrum of predictive and non-predictive outcomes. Further research is needed to explore potential interventions to support colorectal cancer patients with specific disabilities.

In conclusion, the general disability level was not different between colorectal patients with and without a stoma. Patients with a stoma had more limited societal participation, and were more affected by their disease emotionally; however, they were less limited in concentrating on doing a tasks for 10 minutes.

\section{CONFLICT OF INTEREST}

No potential conflict of interest relevant to this article was reported.

\section{ACKNOWLEDGMENTS}

This study was funded by the Basic Science Research Program through a National Research Foundation of Korea (NRF) grant funded by the Ministry of Education, Science and Technology (MEST) (No. 2011-32A-B00142). The authors are indebted to J. Patrick Barron, Professor Emeritus, Tokyo Medical University and Adjunct Professor, Bundang Hospital for his pro bono editing of this manuscript.

\section{REFERENCES}

1. Adderson EE, Boudreaux JW, Cummings JR, Pounds S, Wilson DA, Procop GW, et al. Identification of clinical coryneform bacterial isolates: comparison of biochemical methods and sequence analysis of 16S rRNA and rpoB genes. J Clin Microbiol 2008;46:921-7.

2. Royse CF, Newman S, Chung F, Stygall J, McKay RE, Boldt J, et al. Development and feasibility of a scale to assess postoperative recovery: the post-operative quality recovery scale. Anesthesiology 2010;113:892905.

3. Wilson TR, Alexander DJ. Clinical and non-clinical factors influencing postoperative health-related quality of life in patients with colorectal cancer. Br J Surg 2008;95:1408-15.

4. Engel J, Kerr J, Schlesinger-Raab A, Eckel R, Sauer H, Holzel D. Quality of life in rectal cancer patients: a four-year prospective study. Ann Surg 2003;238:20313.

5. Hohenberger W, Merkel S, Matzel K, Bittorf B, Papadopoulos T, Gohl J. The influence of abdomino-peranal (intersphincteric) resection of lower third rectal carcinoma on the rates of sphincter preservation and locoregional recurrence. Colorectal Dis 2006;8:23-33.

6. Dumont F, Ayadi M, Goere D, Honore C, Elias D. Comparison of fecal continence and quality of life between intersphincteric resection and abdominoperineal resection plus perineal colostomy for ultra-low rectal cancer. J Surg Oncol 2013;108:225-9.

7. Pachler J, Wille-Jorgensen P. Quality of life after rectal resection for cancer, with or without permanent colostomy. Cochrane Database Syst Rev 2012;12:CD004323.

8. Ustun TB, Chatterji S, Kostanjsek N, Rehm J, Kennedy C, Epping-Jordan J, et al. Developing the World Health 
Organization Disability Assessment Schedule 2.0. Bull World Health Organ 2010;88:815-23.

9. Sousa RM, Dewey ME, Acosta D, Jotheeswaran AT, Castro-Costa E, Ferri CP, et al. Measuring disability across cultures: the psychometric properties of the WHODAS II in older people from seven low- and middle-income countries. The 10/66 Dementia Research Group population-based survey. Int J Methods Psychiatr Res 2010;19:1-17.

10. Von Korff M, Crane PK, Alonso J, Vilagut G, Angermeyer MC, Bruffaerts R, et al. Modified WHODAS-II provides valid measure of global disability but filter items increased skewness. J Clin Epidemiol 2008;61: 1132-43.

11. Luciano JV, Ayuso-Mateos JL, Fernandez A, SerranoBlanco A, Roca M, Haro JM. Psychometric properties of the twelve item World Health Organization Disability Assessment Schedule II (WHO-DAS II) in Spanish primary care patients with a first major depressive episode. J Affect Disord 2010;121:52-8.

12. Zhao HP, Liu Y, Li HL, Ma L, Zhang YJ, Wang J. Activity limitation and participation restrictions of breast cancer patients receiving chemotherapy: psychometric properties and validation of the Chinese version of the WHODAS 2.0. Qual Life Res 2013;22:897-906.

13. Yoon JS, Kim JM, Shin IS, Yang SJ, Zheng TJ, Lee HY. Development of Korean version of World Health Organization Disability Assessment Schedule II (WHODAS II-K) in community dwelling elders. J Korean Neuropsychiatr Assoc 2004;43:86-92.

14. World Health Organization. WHO Disability Assessment Schedule 2.0 (WHODAS 2.0) [Internet]. Geneva: World Health Organization; c2017 [cited 2017 Jul 1]. Available from: http://www.who.int/classifications/ icf/whodasii/en/index4.html.

15. Prince M, Glozier N, Sousa R, Dewey M. Measuring disability across physical, mental and cognitive disorders. Arlington: American Psychiatric Publishing Inc.; 2010.

16. Han CW, Lee EJ, Iwaya T, Kataoka H, Kohzuki M. Development of the Korean version of Short-Form 36-
Item Health Survey: health related QOL of healthy elderly people and elderly patients in Korea. Tohoku J Exp Med 2004;203:189-94.

17. Portney LG, Watkins MP. Foundations of clinical research: applications to practice. Upper Saddle River: Prentice-Hall; 2000.

18. Deimling GT, Sterns S, Bowman KF, Kahana B. Functioning and activity participation restrictions among older adult, long-term cancer survivors. Cancer Invest 2007;25:106-16.

19. Neuman HB, Patil S, Fuzesi S, Wong WD, Weiser MR, Guillem JG, et al. Impact of a temporary stoma on the quality of life of rectal cancer patients undergoing treatment. Ann Surg Oncol 2011;18:1397-403.

20. Orsini RG, Thong MS, van de Poll-Franse LV, Slooter GD, Nieuwenhuijzen GA, Rutten HJ, et al. Quality of life of older rectal cancer patients is not impaired by a permanent stoma. Eur J Surg Oncol 2013;39:164-70.

21. Schmidt CE, Bestmann B, Kuchler T, Longo WE, Rohde V, Kremer B. Gender differences in quality of life of patients with rectal cancer: a five-year prospective study. World J Surg 2005;29:1630-41.

22. Sideris L, Zenasni F, Vernerey D, Dauchy S, Lasser P, Pignon JP, et al. Quality of life of patients operated on for low rectal cancer: impact of the type of surgery and patients' characteristics. Dis Colon Rectum 2005;48: 2180-91.

23. Aaronson N, Alonso J, Burnam A, Lohr KN, Patrick DL, Perrin E, et al. Assessing health status and quality-of-life instruments: attributes and review criteria. Qual Life Res 2002;11:193-205.

24. Garin O, Ayuso-Mateos JL, Almansa J, Nieto M, Chatterji S, Vilagut G, et al. Validation of the "World Health Organization Disability Assessment Schedule, WHODAS-2" in patients with chronic diseases. Health Qual Life Outcomes 2010;8:51.

25. Chwastiak LA, Von Korff M. Disability in depression and back pain: evaluation of the World Health Organization Disability Assessment Schedule (WHO DAS II) in a primary care setting. J Clin Epidemiol 2003;56: 507-14. 